\title{
MicroRNA-181a promotes proliferation and inhibits apoptosis by suppressing CFIm25 in osteosarcoma
}

\author{
ZHONG-JIAO ZHU ${ }^{1 *}$, PENG HUANG $^{2 *}$, YAN-XUE CHONG $^{1}$, LI-XIN KANG ${ }^{1}$, \\ XING HUANG ${ }^{3}$, ZHONG-XIU ZHU ${ }^{4}$ and LIN NIE $^{5}$ \\ ${ }^{1}$ Department of Orthopedics, Tengzhou Central People's Hospital, Tengzhou, Shandong 277500; \\ ${ }^{2}$ Department of Emergency, Tengzhou Hospital of Traditional Chinese Medicine, Tengzhou, Shandong 277500; \\ ${ }^{3}$ Department of Pharmacy Intravenous Admixture Service, Tengzhou Central People's Hospital, Tengzhou, Shandong 277500; \\ ${ }^{4}$ Department of Oncology, Affiliated Hospital of Shandong Academy of Medical Sciences, Jinan, Shandong 250031; \\ ${ }^{5}$ Department of Orthopedics, Qilu Hospital of Shandong University, Jinan, Shandong 250012, P.R. China
}

Received July 19, 2015; Accepted August 22, 2016

DOI: $10.3892 / \mathrm{mmr} .2016 .5741$

\begin{abstract}
MicroRNA-181a (miR-181a) is upregulated in osteosarcoma, and its overexpression promotes the proliferation and inhibits the apoptosis of osteosarcoma cells. However, the mechanism of miR-181a as an oncogene remains to be fully elucidated in osteosarcoma. Cleavage factor (CF) Im25 links alternative polyadenylation to glioblastoma tumor suppression, however, its role in osteosarcoma has not been reported. In the present study, it was confirmed that the expression of miR-181a was upregulated in osteosarcoma, and that silencing miR-181a inhibited the proliferation and promoted the apoptosis of osteosarcoma cells. miRNAs are short non-coding RNAs, which regulate target $\mathrm{mRNAs}$ by binding predominantly to the 3'untranslated region (3'UTR), inducing either translational repression or degradation of the target. In the present study, target genes of miR-181a were screened using miRanda, which is a commonly used prediction algorithm. It was found that miR-181a targeted the 3'UTR of CFIm25 mRNA. Subsequent experiments confirmed that miR-181a downregulated the expression of CFIm 25 in osteosarcoma cells. Finally, it was demonstrated that the CFIm 25 protein was also downregulated in osteosarcoma tissues, and inhibited the proliferation and promoted the apoptosis of the cells. Elucidating the roles of miR-181a and CFIm25 in osteosarcoma not only assists in further understanding the pathogenesis and progression of this disease, but also offers novel targets for effective therapies.
\end{abstract}

Correspondence to: Dr Lin Nie, Department of Orthopedics, Qilu Hospital of Shandong University, 107 Wen Hua Xi Road, Jinan, Shandong 250012, P.R. China

E-mail: nielin633@yahoo.com

*Contributed equally

Key words: microRNA-181a, cleavage factor Im25, osteosarcoma

\section{Introduction}

Osteosarcoma, the most common primary malignant tumor of bone, has an annual worldwide incidence of between one and three cases per 1,000,000, and arises primarily in children and adolescents, with a second peak in incidence in those $>50$ years old (1-6). Although osteosarcoma has been treated with surgery and chemotherapy for $>30$ years, patients with recurrent or metastatic osteosarcoma have a poor prognosis (7-11). Further elucidating the molecular mechanism of the disease not only enables further understanding of the pathogenesis and progression of the disease, but also assists in identifying novel targets for effective therapies.

It has become increasingly recognized that mRNA $3^{\prime}$ end formation is subject to dynamic regulation under diverse physiological conditions (12-15) and the processing is crucial for mRNA maturation in eukaryotic cells, promoting mRNA stability, efficient nuclear transport and translation (16-18). This process involves six primary protein complexes in mammals: The cleavage and polyadenylation specificity factor, cleavage stimulatory factor, cleavage factor I and II (CFIm and CFIIm), poly(A) polymerase and poly(A) binding protein, together with RNA polymerase II $(19,20)$ and additional proteins $(21)$. The downregulation of CFIm 25 in glioblastoma cells enhances their tumorigenic properties and increases tumor size, whereas the overexpression of CFIm 25 reduces these properties and inhibits tumor growth (22). In addition, the global shortening of mRNAs through alternative polyadenylation (APA), which occurs during enhanced cellular proliferation, represents an important mechanism of regulated gene expression, and a subset of CFIm25-regulated APA genes with shortened 3'untranslated regions (UTRs) have been identified in glioblastoma tumors with reduced expression of CFIm25 $(12,22,23)$. These findings identified a pivotal role of CFIm 25 in governing APA, however, the role of CFIm 25 in osteosarcoma remains to be elucidated.

MicroRNAs (miRNAs/miRs) are a class of non-coding RNAs, which are able to regulate gene expression at the post-transcriptional level by binding to the 3'UTR of target mRNAs through partial sequence homology, and inhibiting 
translation and/or mRNA degradation (24-26). Aberrant miRNA expression has been linked to osteosarcoma (27-29), and it has been found to be involved in several cellular processes, including proliferation, differentiation, migration and apoptosis (30-35). miRNA-181a is upregulated in osteosarcoma. It facilitates the proliferation and invasion, and suppresses the apoptosis of osteosarcoma cells. However, the mechanism of miR-181a as an oncogene in osteosarcoma remains to be fully elucidated.

In the present study, it was first confirmed that the expression of miR-181a was upregulated in osteosarcoma, compared with adjacent normal tissues. Silencing miR-181a inhibited the proliferation and promoted the apoptosis of osteosarcoma cells. Target genes of miR-181a were screened using miRanda, which is a commonly used prediction algorithm. It was found that miR-181a targeted the 3'UTR of CFIm25 mRNA. Subsequent experiments confirmed that miR-181 downregulated the expression of CFIm 25 in osteosarcoma cells. Finally, the present study demonstrated that CFIm 25 protein was downregulated in osteosarcoma tissues, in addition to inhibiting proliferation and promoting apoptosis in the cells.

\section{Materials and methods}

Cell culture and expression plasmids. MG63 human osteosarcoma cells (American Type Culture Collection, Rockville, MD USA) were maintained in minimum essential medium (Life Technologies; Thermo Fisher Scientific, Inc., Waltham, MA, USA) containing 10\% fetal bovine serum (Biowest, Riverside, MO, USA), $1 \%$ non-essential amino acids, and $1 \%$ penicillin/streptomycin in a $5 \% \mathrm{CO}_{2}$ incubator at $37^{\circ} \mathrm{C}$. The expression plasmid CFIm 25 and empty vector (pcDNA.3.1) were purchased from Tiangene (Tianjin, China). In the transfection experiments, the cells were cultured at a density of $2 \times 10^{5}$ in serum-free medium without antibiotics at $60 \%$ confluence for $24 \mathrm{~h}$ in a $5 \% \mathrm{CO}_{2}$ incubator at $37^{\circ} \mathrm{C}$, and then transfected with transfection reagent (Lipofectamine 2000; Invitrogen; Thermo Fisher Scientific, Inc.), according to manufacturer's protocol. Following incubation for $6 \mathrm{~h}$, the medium was removed and replaced with normal culture medium for $48 \mathrm{~h}$ in a $5 \% \mathrm{CO}_{2}$ incubator at $37^{\circ} \mathrm{C}$.

miRNA precursors and anti-miRNA oligonucleotides. The locked nucleic acid-modified oligonucleotide inhibitor (anti-miR-181a) used for miRNA knockdown and the scramble control were purchased from Exiqon, Inc. (Woburn, MA, USA).

Reverse transcription-quantitative polymerase chain reaction $(R T-q P C R)$ analysis of miRNA. Total RNA, including miRNA from tissue samples and cells was isolated using TRIzol reagent (Invitrogen; Thermo Fisher Scientific, Inc.). The concentration of RNA was measured using a NanoDrop ND-1000 spectrophotometer (NanoDrop Technologies; Thermo Fisher Scientific, Inc.). The mRNA was reverse transcribed using a reverse transcription kit (Takara Biotechnology Co., Ltd., Dalian, China), according to the manufacturer's protocol. The relative levels of miR-181a were examined using altered stem-loop RT-PCR with specific RT and PCR primers. U6 served as a control. The reverse transcription primers for miR-181a and U6 are as follows: RT primer 5'-GTCGTATCC
AGTGCAGGGTCCGAGGTATTCGCACTGGATACGACA CTCACCGA-3', specific primer 5'-GAACATTCAACGCTG TCGGTG-3' and U6 5'-ATCCAGTGCAGGGTCCGA GGTA-3'. Relative quantification of mRNA expression levels was performed according to the manufacturer's instructions (Bio Rad, Hercules, CA, USA). Detection of the mature form of the miRNAs was performed using an mirVana qRT-PCR miRNA detection kit and primer sets, according to the manufacturer's protocol (Ambion; Thermo Fisher Scientific, Inc.). U6 small nuclear RNA was used as an internal control.

Colony formation. Colony formation analysis was performed as described previously (36). Briefly, when the transfected cells were growing in the logarithmic phase they were trypsinized with $0.05 \%$ Trypsin-EDTA (Gibco; Thermo Fisher Scientific, Inc.) and seeded into 6-well plates at a density of 2,000 cells/well. The cells were maintained in an incubator at $37^{\circ} \mathrm{C}$ for 7 days. On day 8 , the colonies were washed with phosphate-buffered saline (PBS), fixed with formalin (10\%), and stained with methyl violet, all were purchased from Beyotime Institute of Biotechnology (Haimen, China). The methyl violet dye was then washed with PBS and the number of colonies were counted under a microscope (IX53; Olympus Corporation, Tokyo, Japan). The following calculations were then performed: Colony inhibition rate $=[(1-$ number of colonies in experimental groups) / control group] x 100\%; and colony forming efficiency $=1$ colony inhibition rate.

\section{3-(4,5-Dimethyl-2-thiazolyl)-2,5-diphenyl-2H-tetrazolium}

bromide (MTT) assay. The analysis of proliferation was performed using an MTT assay as described previously (37). Cell viability was determined by MTT assay. Briefly, transfected cells were seeded into 96-well plates at a density of $1.0 \times 10^{5}$ cells/well and $10 \mu 1$ MTT (concentration, $5 \mathrm{mg} / \mathrm{ml}$; Sigma Aldrich, St Louis, MO, USA) was added into $100 \mu \mathrm{l}$ medium at indicated time points. The cells were incubated with MTT for $\sim 4 \mathrm{~h}$ at $37^{\circ} \mathrm{C}$, followed by removal of MTT and addition of $150 \mu \mathrm{l}$ DMSO. Following incubation with DMSO for $10 \mathrm{~min}$ in the dark, absorbance was measured at $570 \mathrm{~nm}$ (A570nm) with a microplate reader (Biorad-168-1000XC; Bio-Rad Laboratories, Hercules, CA, USA).

Western blot analysis. The cells were seeded into 6-well plate at a density of $3.0 \times 10^{5}$ cells/well and the cells were transfected when the cell density reached $\sim 80 \%$ confluency in the second day. At $48 \mathrm{~h}$ following transfection, the cells were lysed using RIPA buffer for $30 \mathrm{~min}$ at $4^{\circ} \mathrm{C}$. The protein concentration was measured by the BCA method. Western blot analysis was performed as described previously (38). Briefly, following incubation with primary antibody anti-BCL2 (cat. no. ab32124; 1:500; Abcam, Cambridge, MA, USA), anti-myeloid cell leukemia 1 (MCL1) (cat. no. ab32087; 1:500; Abcam, Cambridge, MA, USA), anti-c-myc (cat. no. ab32072; 1:500; Abcam), anti-p53 (cat. no. ab1431; 1:500; Abcam), anti-Ki-67 (cat. no. ab16667; 1:500; Abcam), anti-proliferating cell nuclear antigen (PCNA; cat. no. ab1431; 1:500; Abcam), anti-cyclin D1 (cat. no. EPR2241; 1:500; Abcam) and anti- $\beta$-actin (cat. no. ab5694; 1:500; Abcam) overnight at $4^{\circ} \mathrm{C}$, incubation with IRDyeTM-800 conjugated anti-rabbit secondary antibodies (caat. no. 925-32211; Li-COR 
Biosciences, Lincoln, NE, USA) was performed for $30 \mathrm{~min}$ at room temperature. The specific proteins were visualized using an Odyssey ${ }^{\mathrm{TM}}$ infrared imaging system (Gene Company, Ltd., Lincoln, NE, USA).

Methods of bioinformatics analysis. The analysis of potential miR target sites was performed using the commonly used prediction algorithm, miRanda (http://www.microrna. org/microrna/home.do).

Immunofluorescence analysis. For the immunofluorescence analysis of MG63 human osteosarcoma cells. The cells were plated on glass coverslips in 6-well plates at density of $3.0 \times 10^{5}$ cells/well and transfected with $30 \mathrm{nM}$ anti-miR-181a or the scramble control. At $36 \mathrm{~h}$ post-transfection, the coverslips were stained with anti-CFIm 25 antibodies. Alexa Fluor 488 goat anti-mouse IgG antibody or goat anti-rabbit IgG antibody were used as secondary antibodies (Invitrogen; Thermo Fisher Scientific, Inc.). The coverslips were counterstained with DAPI (Invitrogen; Thermo Fisher Scientific, Inc.) for the visualization of nuclei. Microscopic analysis was performed with a confocal laser-scanning microscope (Leica Microsystems, Bensheim, Germany). Fluorescence intensities were measured in a number of areas with 200-300 cells per coverslip, and analyzed using ImageJ $1.37 \mathrm{v}$ software (http://rsb.info.nih. gov/ij/index.html).

RT-qPCR analysis of CFIm25. Total RNA was isolated from the cells using TRIzol reagent (Invitrogen; Thermo Fisher Scientific, Inc.). First-strand cDNA was synthesized from the total RNA using M-MLV reverse transcriptase (Promega, Madison, WI, USA) and random hexamer primers (Sangon Biotech, Shanghai, China). The thermal cycle profile was as follows: Denaturation for $30 \mathrm{sec}$ at $95^{\circ} \mathrm{C}$, annealing for $45 \mathrm{sec}$ at $52-58^{\circ} \mathrm{C}$ depending on the primers used, and extension for $45 \mathrm{sec}$ at $72^{\circ} \mathrm{C}$. The PCR products were visualized on $2 \%$ agarose gels stained with ethidium bromide under UV transillumination. RT-qPCR was performed using Power SYBR Green PCR Master Mix (Applied Biosystems; Thermo Fisher Scientific, Inc.) according to the manufacturer's protocol. The primer sequences for CFIm25 were: Forward (F) 5'-AGTGTTTACAGGCACAAGAGG-3' and reverse (R) 5'-TTCAGGAACAGGCAAGGA-3. GAPDH primers were: F 5'-GAAGGTCGGAGTCAACGGATTTG-3' and R 5'-ATGGCATGGACTGTGGTCATGAG-3'. The products were then separated by electrophoresis using $1.5 \%$ agarose gels. The bands were visualized using the BioSpectrum 410 multispectral imaging system with a Chemi HR camera 410 (UVP, Upland, CA, USA) and quantified as previosly described (39).

TUNEL staining. For the analysis of apoptosis, TUNEL staining was performed as described previously (40). The TUNEL assays were performed with the TMR red In Situ Cell Death Detection kit (supplemented with $0.1 \%$ sodium citrate; Hoffmann La Roche Inc., Nutley, NJ, USA), according to the manufacturer's instructions.

Statistical analysis. Data are presented as the mean \pm standard error of the mean. Student's t-test (two-tailed) was used to compare between the two groups. Statistical analysis was performed using SPSS version 17 (SPSS, Inc., Chicago, IL, USA). $\mathrm{P}<0.05$ was considered to indicate a statistically significant difference.

\section{Results}

Expression of miR-181a is upregulated in osteosarcoma tissues, promoting the proliferation and inhibiting the apoptosis of osteosarcoma cells. In order to identify and compare the expression levels of miR-181a between osteosarcoma tissues and adjacent normal tissues, RT-qPCR was performed on the cancer tissues and normal tissues. The RT-qPCR analysis revealed that the expression of miR-181a in six osteosarcoma tissue samples were significantly higher, compared with those of the adjacent normal tissues (Fig. 1A). These data suggested that miR-181a is an oncogene in osteosarcoma.

To further identify the role of miR-181a in osteosarcoma cells, the MG63 osteosarcoma cells were transfected with anti-miR-181a. Following stable transfection, the expression of miR-181a was detected using RT-qPCR analysis, and colony formation of the MG63 cells was assessed using a colony formation assay. The results showed that exogenous anti-miR-181a significantly downregulated the expression of miR-181a in the MG63 cells (Fig. 1B) and silencing miR-181a significantly decreased the colony formation rate of the MG63 cells (Fig. 1C). In addition, MTT assays were performed to detect the proliferation of the MG63 cells transfected with anti-miR-181a and the scramble control. The results showed that anti-miR-181a inhibited the proliferation of the MG63 cells following $48 \mathrm{~h}$ of transfection, compared with the scramble-transfected cells (Fig. 1D). In subsequent experiments, western blot analysis was used to identify whether the proteins of proliferation-associated markers were also affected by silencing miR-181a in the cells. The results showed that the protein expression levels of c-myc, PCNA and cyclin D1 were downregulated, and the expression of p53 was upregulated by anti-miR-181a in the cells (Fig. 1E).

On demonstrating that silencing miR-181a inhibited the proliferation of the MG63 cells, to provide further evidence that miR-181a was involved in the regulation of MG63 cell apoptosis, a TUNEL assay was performed to analyze whether silencing miR-181a affected apoptosis in the MG63 cells. The TUNEL assay revealed a change in the apoptotic rate of the MG63 cells transfected with anti-miR-181a. Specifically, silencing miR-181a promoted apoptosis in the MG63 cells (Fig. 1F).

Western blot analysis was also performed to identify whether proteins of apoptosis-associated markers were also affected by miR-181a in the cells. Bcl-2 and MCL1 are important anti-apoptotic molecules $(41,42)$. The results showed that the expression levels of BCL2 and MCL1 were downregulated by anti-miR-181a in the cells (Fig. 1E).

Silencing miR-181a restores the protein expression of CFIm 25 in osteosarcoma. miRNAs/miRs are a class of non-coding RNAs, which are able to regulate gene expression at the post-transcriptional level by binding to the 3'UTR 
A

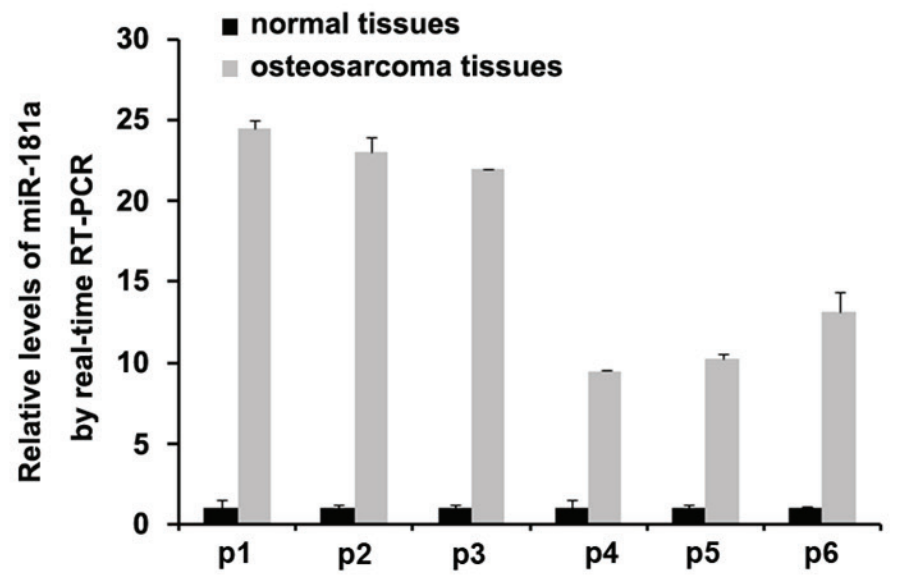

B

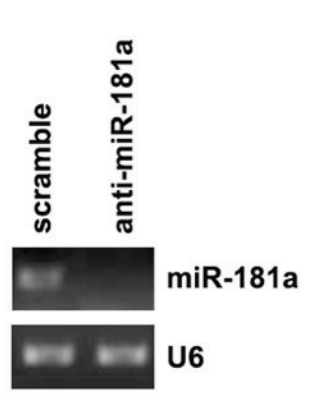

$\mathbf{E}$

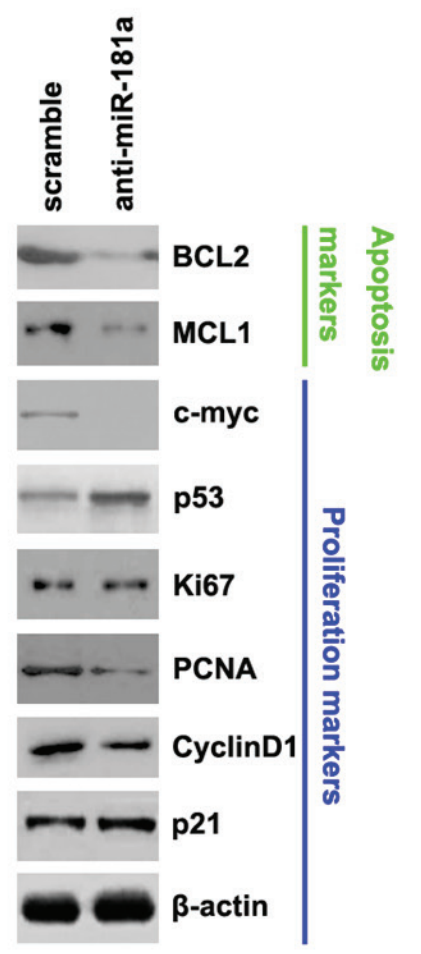

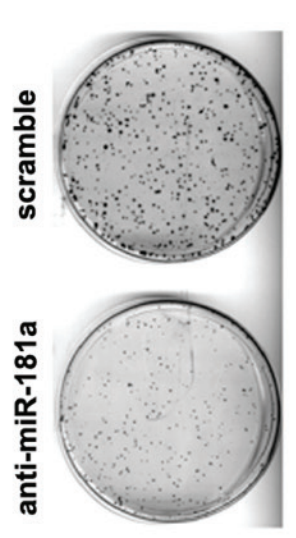

C scramble

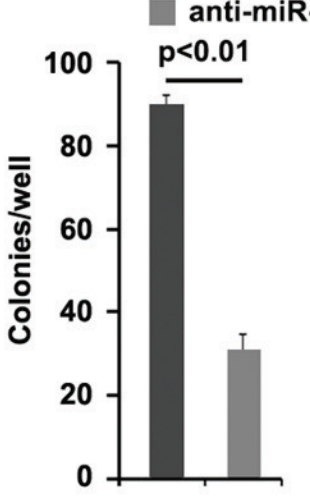

F

F
D

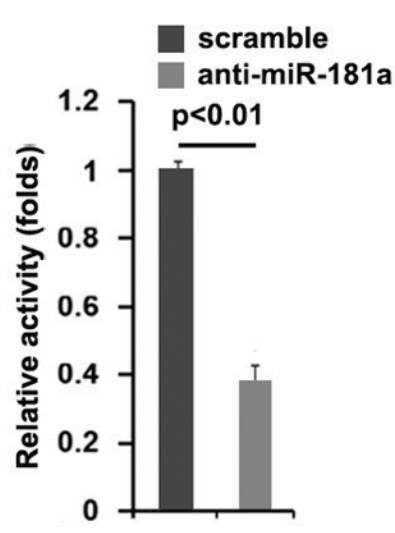

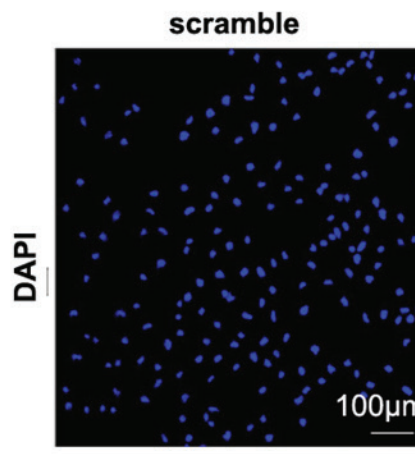

anti-miR-181a
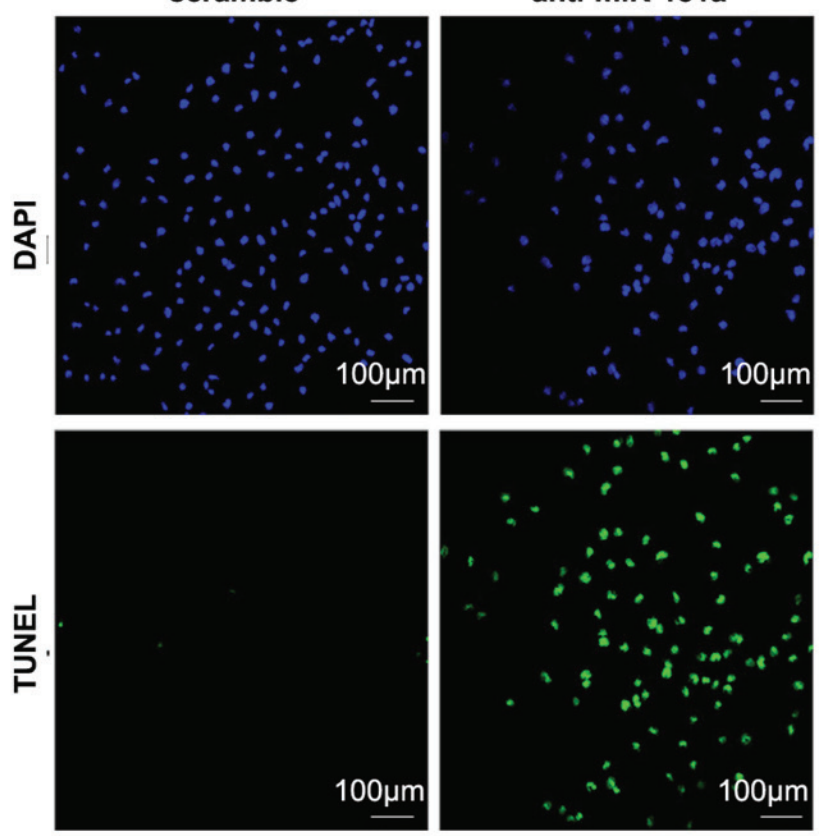

Figure 1. Expression of miR-181a is upregulated in osteosarcoma tissues, and its silencing inhibits proliferation and promotes apoptosis. RT-qPCR analysis was used to compare miR-181a between (A) osteosarcoma tissues and adjacent normal tissues (n=6) and between (B) MG63 cells transfected with scramble and anti-miR-181a ( $\mathrm{n}=3)$. U6 was used as a loading control. (C) Colony formation assay of MG63 cells transfected with scramble and anti-miR-181a. Colonies of $>50$ cells were counted (n=3). (D) 3-(4,5-Dimethyl-2-thiazolyl)-2,5-diphenyl-2H-tetrazolium bromide assay (n=3) and (E) Western blot analysis for BCL2, MCL1, c-myc, p53, Ki-67, PCNA, cyclin D1 and p21 in MG63 cells transfected with scramble and anti-miR-181a. $\beta$-actin was used as a loading control (n=3). (F) TUNEL assay ( $n=3$ ) for MG63 cells transfected with scramble and anti-miR-181a. miR, microRNA; RT-qPCR, reverse transcription-quantitative polymerase chain reaction; BCL2, B cell lymphoma 2; MCL1, myeloid cell leukemia 1; PCNA, proliferating cell nuclear antigen. 
$\mathbf{A}$

C hsa-miR-181a/NUDT21 alignment
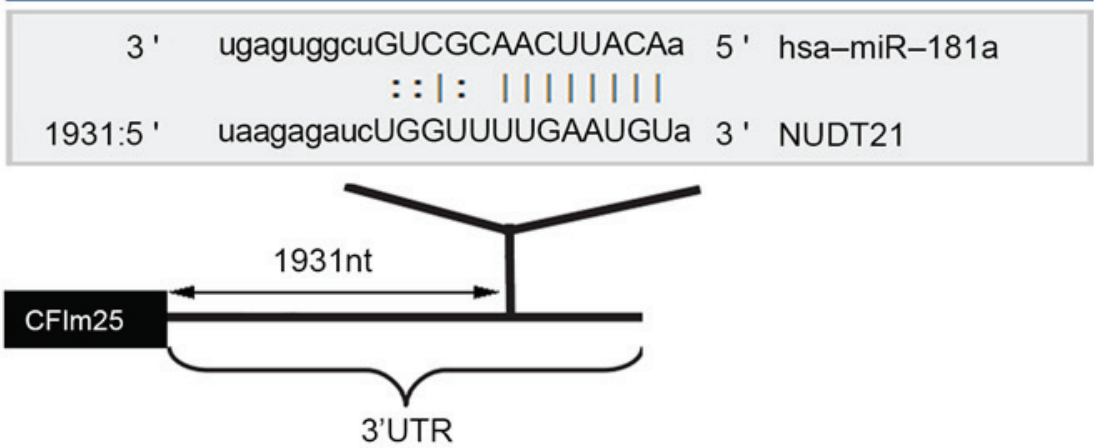

C
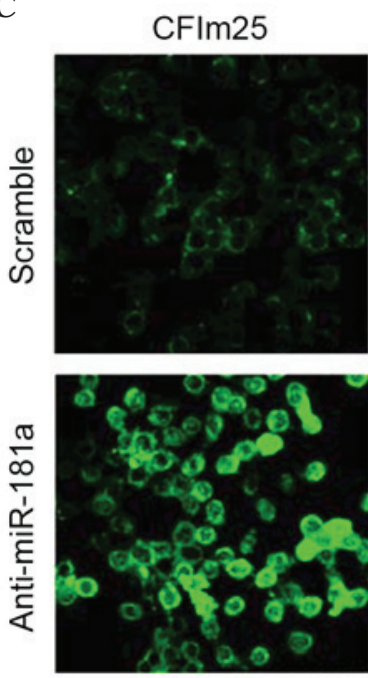

D

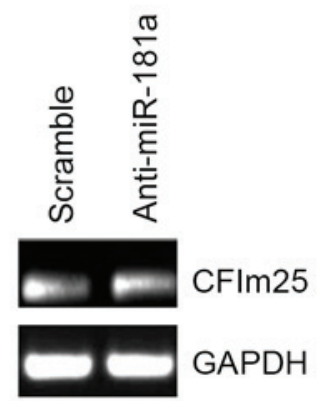

$\mathbf{E}$
B

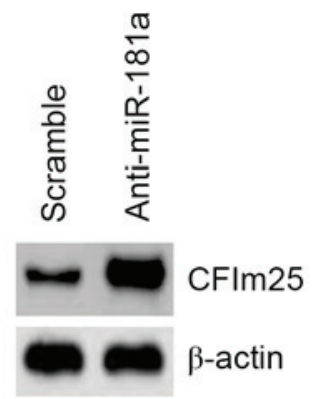

Scramble

Anti-miR-181a
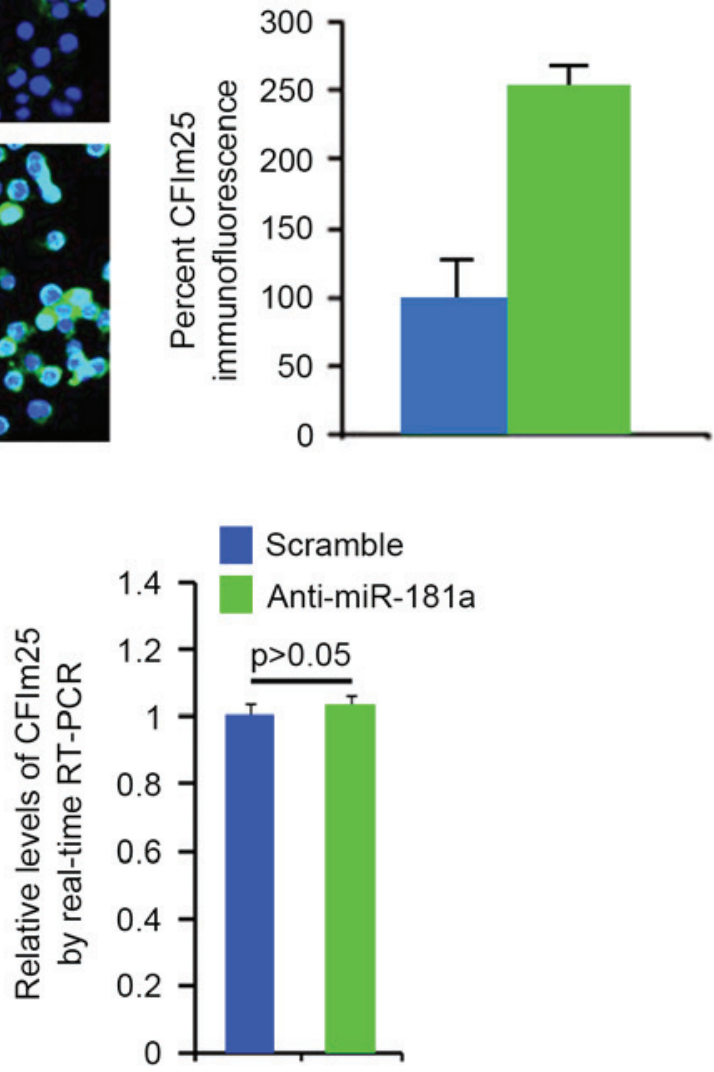

Figure 2. anti-miR-181a restores the protein expression of CFIm25 in MG63 cells. (A) Schematic of predicted miR-181a-binding sites in the 3'untranslated region of CFIm25 mRNA. (B) Western blot analysis of the protein levels of CFIm25 in MG63 cells transfected with scramble and anti-miR-181a. $\beta$-actin was used as a loading control ( $n=3$ ). (C) Immunofluorescence analyses of MG63 cells transfected with scramble and anti-miR-181a; (left) microscopic images of immunofluorescence staining of one representative experiment (magnification, x100); (right) graph of mean fluorescence intensities of three independent experiments. (D and E) RT-qPCR analysis of the mRNA expression of CFIm25 in MG63 cells transfected with scramble or anti-miR-181a. GAPDH was used as a loading control $(n=3)$. miR, microRNA; CFIm25, CFm25, cleavage factor m25; RT-qPCR, reverse transcription-quantitative polymerase chain reaction.

of target mRNAs through partial sequence homology; this inhibits translation and/or mRNA degradation (24-26). The upregulation of specific miRNAs can contribute to the downregulation of tumor suppressive genes $(31,43,44)$. Thus, the present study hypothesized that miR-181a affected proliferation and apoptosis by regulating target genes.
In the present study, targeted genes of miR-181a were screened using TargetScan (http://www.microrna. org/microrna/home.do), and a large number of target genes were identified. The gene of interest, CFIm25, was used as it has been regarded as a tumor suppressor gene in malignant tumors (22). Target sites on the 3'UTR of CFIm25 are shown 
A

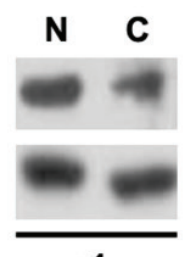

p1

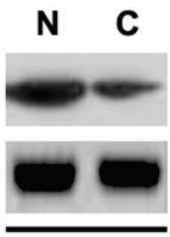

p4

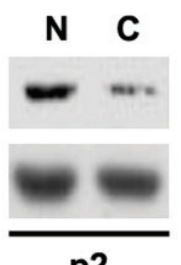

p2

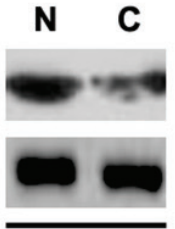

p5

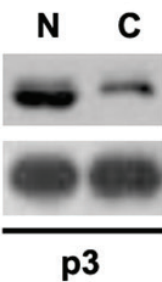

N C

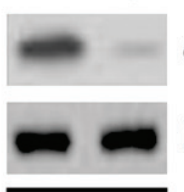

p6

C
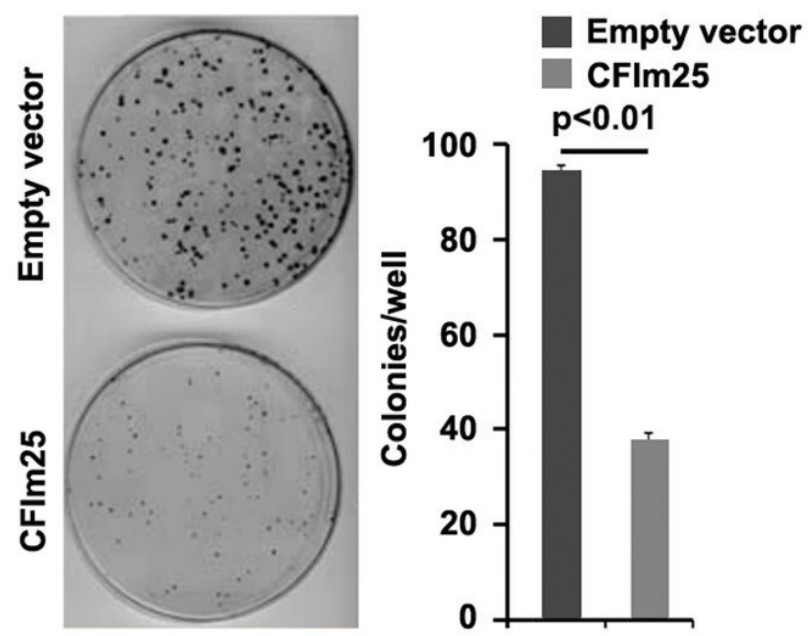

B

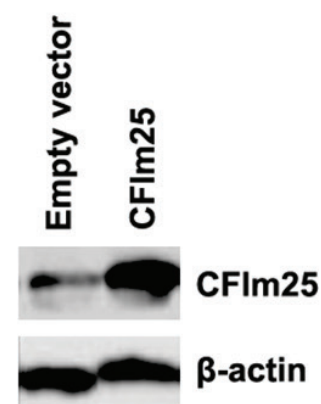

D
CFIm25

$\beta$-actin

CFIm25

$\beta$-actin

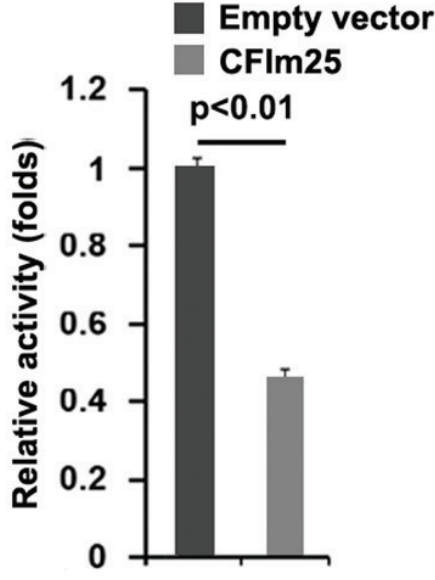

$\mathbf{E}$

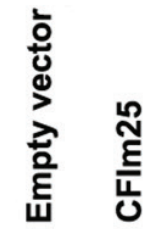

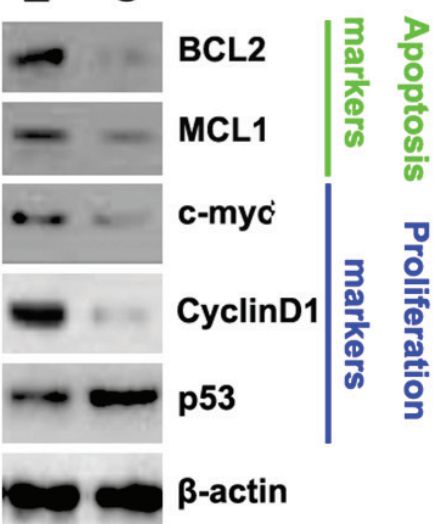

F

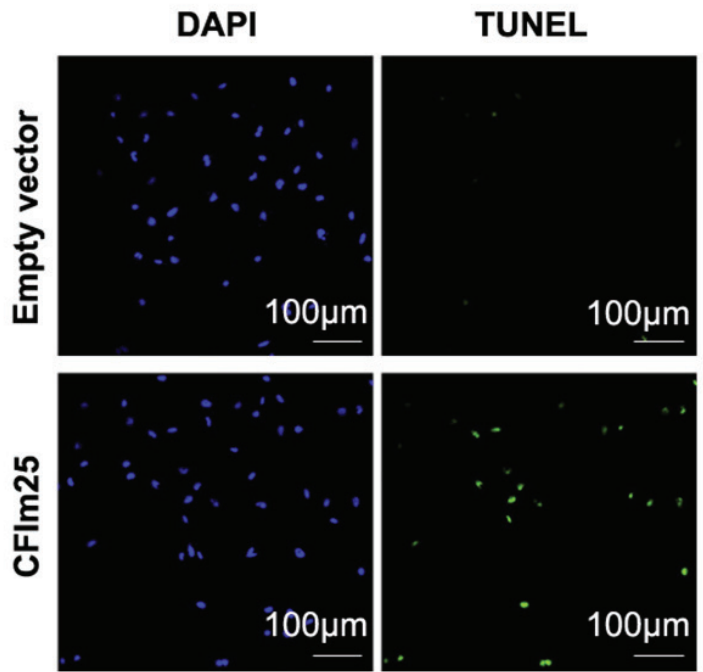

Figure 3. CFIm25 inhibits proliferation and promotes apoptosis of MG63 cells. (A) Western blot analysis for the protein expression of CFIm25 in osteosarcoma tissues. $\beta$-actin was used as a loading control $(n=3)$. (B) Western blot analysis for the protein expression of CFIm25 in MG63 cells transfected with empty vectors or CFIm25-expressing plasmids. $\beta$-actin was used as a loading control ( $\mathrm{n}=3$ ). (C) Colony formation assay for MG63 cells transfected with empty vectors or CFIm25-expressing plasmids. Colonies of $>50$ cells were counted (n=3). (D) 3-(4,5-Dimethyl-2-thiazolyl)-2,5-diphenyl-2H-tetrazolium bromide assay for MG63 cells transfected with empty vectors and CFIm25-expressing plasmids ( $\mathrm{n}=3$ ). (E) Western blot analysis for the protein expression levels of BCL2, MCL1, c-myc, cyclin D1 and p53 in MG63 cells transfected with scramble and anti-miR-181a. $\beta$-actin was used as a loading control (n=3). (F) TUNEL assay for MG63 cells transfected with empty vectors and CFIm25-expressing plasmids ( $\mathrm{n}=3$ ). miR, microRNA; CFIm25, cleavage factor Im25; BCL2, B cell lymphoma 2; MCL1, myeloid cell leukemia 1. 
in Fig. 2A. Therefore, miR-181a may downregulate the expression of CFIm 25 by targeting its 3'UTR in osteosarcoma cells.

Western blot analyses was performed on MG63 cells transfected with anti-miR-181c or scramble. The results confirmed that the protein level of CFIm25 was increased in the cells transfected with anti-miR-181a (Fig. 2B). Consistent with the results of western blot analyses, immunofluorescence analyses demonstrated that the protein level of CFIm 25 increased in MG63 cells transfected with anti-miR-181a, compared with scramble-transfected groups (Fig. 2C). To identify whether CFIm25 mRNA was increased by the silencing of miR-181a, RT-qPCR analysis was performed to detect the mRNA expression of CFIm25 in theMG63 cells transfected with anti-miR-181a or scramble. The results of the RT-qPCR analysis demonstrated that silencing of miR-181a had no effect on the mRNA expression of CFIm25 in the MG63 cells (Fig. 2D and $\mathrm{E})$.

CFIm25 protein inhibits proliferation and promotes apoptosis in MG63 osteosarcoma cells. The observation that silencing miR-181a inhibited proliferation, promoted apoptosis and upregulated the expression of CFIm25 in the MG63 cells cells, indicated that anti-miR-181a inhibited proliferation and promoted apoptosis via upregulating the expression of CFIm25. Thus, the present study investigated the roles of CFIm25 in osteosarcoma.

To assess the expression of CFIm25 in osteosarcoma, western blot analysis was performed on six pairs of osteosarcoma tissues and matched adjacent normal tissue samples. The expression of CFIm25 was consistently lower in the osteosarcoma tissues, compared with the normal tissues (Fig. 3A). Subsequently, CFIm25-expressing plasmids were used, and the roles of CFIm25 in MG63 cell were investigated. In order to demonstrate that CFIm25-expressing plasmids stably upregulated the protein expression of CFIm25, western blot analysis was performed on MG63 cells transfected with CFIm25-expressing plasmids. The results showed that the protein expression of CFIm 25 was significantly increased by the CFIm25-expressing plasmids in the cells (Fig. 3B). To determine the roles of CFIm 25 in colony formation ability and proliferation of the MG63 cells, colony formation and MTT assays were performed. The results of the colony formation assay demonstrated that CFIm25 suppressed colony formation in the MG63 cells (Fig. 3C) and, consistent with the colony formation assay, the MTT assay showed that CFIm25 inhibited the proliferation of the cells (Fig. 3D). In subsequent experiment, we performed western blot analysis to identify whether the proteins of proliferation-associated markers were also affected by CFIm 25 in the cells. The results of the present study showed that the expression of cyclin D1 was downregulated the expression of p53, were upregulated by CFIm 25 in the cells (Fig. 3E).

Having demonstrated that overexpressing CFIm 25 inhibited the proliferation in MG63 cells, to provide further evidence that CFIm25 was involved in the regulation of MG63 cell apoptosis, a TUNEL assay was performed to analyze whether overexpressing CFIm25 affected apoptosis in MG63 cells. The TUNEL assay revealed a change in the apoptotic rate of the MG63 cells transfected with CFIm25. Specifically, overexpressing CFIm25 promoted apoptosis in the MG63 cells (Fig. 3F).
Western blot analysis was also performed to identify whether the protein levels of apoptosis-associated markers were also affected by CFIm 25 in the cells. The results of the western blot analysis showed that the expression levels of BCL2 and MCL1 were downregulated in the CFIm25-overexpressing cells (Fig. 3E).

\section{Discussion}

Osteosarcoma is the most common primary sarcoma of bone and is a leading cause of cancer-associated mortality among adolescents and young adults (3). The cellular events, which initiate and propagate osteosarcomagenesis remain to be fully elucidated (45). miRNAs are short noncoding RNAs, which post-transcriptionally modify gene expression in eukaryotic cells (46). The expression of a single miRNA can silence a large number of genes, enabling these molecules to have extensive control over several cellular functions (46). Knowledge of individual miRNAs affecting developmental biology, cellular differentiation programs and oncogenesis continues to increase. miR-181a is upregulated in osteosarcoma, can facilitate proliferation and invasion, and suppress apoptosis in osteosarcoma cells (47). Consistent with a previous report (47), the present study found that the expression of miR-181a is upregulated in osteosarcoma tissues, and that silencing it can inhibit proliferation and promote apoptosis in osteosarcoma cells.

The global shortening of mRNAs through APA, which occurs during enhanced cellular proliferation, represents a vital mechanism of regulated gene expression, which remains to be fully elucidated $(12,23)$. The 3'UTR truncation of growth-promoting mRNA transcripts, which can relieve intrinsic miRNA-mediated repression has been observed to correlate with cellular transformation (13). CFIm25, is a broad repressor of proximal poly(A) site usage and, when depleted, increases cell proliferation (47). Marked increases in the expression of several known oncogenes, including cyclin D1, are observed as a consequence of CFIm25 depletion (47). Consistent with this previous report, the present study found that overexpressing CFIm25 suppressed proliferation, promoted apoptosis and inhibited the expression of cyclin D1 in osteosarcoma cells. It was also demonstrated that the protein expression of CFIm 25 was restored by silencing miR-181a in the osteosarcoma cells.

Elucidating the mechanism by which silencing miR-181a inhibits proliferation and promotes apoptosis by restoring CFIm 25 improves understanding of the molecular mechanisms underlying proliferation and apoptosis in osteosarcoma. The suppression of miR-181a may represent a promising therapeutic strategy to restore the CFIm25-mediated regulation of proliferation and apoptosis. However, the roles of miR-181a and CFIm25 require further confirmation in vivo.

\section{References}

1. Sweetnam R: Osteosarcoma. Br J Hosp Med 28: 112, 116-121, 1982.

2. Mirabello L, Troisi RJ and Savage SA: Osteosarcoma incidence and survival rates from 1973 to 2004: Data from the surveillance, epidemiology, and end results program. Cancer 115: 1531-1543, 2009.

3. Ottaviani G and Jaffe N: The epidemiology of osteosarcoma. Cancer Treat Res 152: 3-13, 2009. 
4. Damron TA, Ward WG and Stewart A: Osteosarcoma, chondrosarcoma, and Ewing's sarcoma. National Cancer Data Base report. Clin Orthop Relat Res 459: 40-47, 2007.

5. Dorfman HD and Czerniak B: Bone cancers. Cancer 75 (1 Suppl): S203-S210, 1995.

6. Geller DS and Gorlick R: Osteosarcoma: A review of diagnosis, management, and treatment strategies. Clin Adv Hematol Oncol 8: 705-718, 2010.

7. Bielack SS, Marina N, Ferrari S, Helman LJ, Smeland S, Whelan JS and Reaman GH: Osteosarcoma: The same old drugs or more? J Clin Oncol 26: 3102-3103; author reply 3104-3105, 2008.

8. Chou AJ, Geller DS and Gorlick R: Therapy for osteosarcoma: Where do we go from here? Paediatr Drugs 10: 315-327, 2008.

9. O'Day K and Gorlick R: Novel therapeutic agents for osteosarcoma. Expert Rev Anticancer Ther 9: 511-523, 2009.

10. Bernthal NM, Federman N, Eilber FR, Nelson SD, Eckardt JJ, Eilber FC and Tap WD: Long-term results ( $>25$ years) of a randomized, prospective clinical trial evaluating chemotherapy in patients with high-grade, operable osteosarcoma. Cancer 118 : 5888-5893, 2012.

11. Link MP, Goorin AM, Miser AW, Green AA, Pratt CB, Belasco JB, Pritchard J, Malpas JS, Baker AR, Kirkpatrick JA, et al: The effect of adjuvant chemotherapy on relapse-free survival in patients with osteosarcoma of the extremity. N Engl J Med 314: 1600-1606,1986.

12. Sandberg R, Neilson JR, Sarma A, Sharp PA and Burge CB Proliferating cells express mRNAs withshortened 3' untranslated regions and fewer microRNA target sites. Science 320: 1643-1647, 2008.

13. Mayr C and Bartel DP: Widespread shortening of 3'UTRs by alternative cleavage and polyadenylation activates oncogenes in cancer cells. Cell 138: 673-684, 2009.

14. Ji Z, Lee JY, Pan Z, Jiang B and Tian B: Progressive lengthening of 3' untranslated regions of mRNAs by alternative polyadenylation during mouse embryonic development. Proc Natl Acad Sci USA 106: 7028-7033, 2009.

15. Mangone M,Manoharan AP,Thierry-MiegD, Thierry-Mieg J,Han T, Mackowiak SD, Mis E, Zegar C, Gutwein MR, Khivansara V, et al The landscape of C. elegans 3'UTRs. Science 329: 432-435, 2010.

16. Jacobson A and Peltz SW: Interrelationships of the pathways of mRNA decay and translation in eukaryotic cells. Annu Rev Biochem 65: 693-739, 1996.

17. Wickens M, Anderson P and Jackson RJ: Life and death in the cytoplasm: Messages from the 3' end. Curr Opin Genet Dev 7: 220-232, 1997.

18. Garneau NL, Wilusz J and Wilusz CJ: The highways and byways of mRNA decay. Nat Rev Mol Cell Biol 8: 113-126, 2007.

19. McCracken S, Fong N, Rosonina E, Yankulov K, Brothers G, Siderovski D, Hessel A, Foster S, Shuman S and Bentley DL: 5'-Capping enzymes are targeted to pre-mRNA by binding to the phosphorylated carboxy-terminal domain of RNA polymerase II Genes Dev 11: 3306-3318, 1997.

20. Hirose Y and Manley JL: RNA polymerase II is an essential mRNA polyadenylation factor. Nature 395: 93-96, 1998.

21. Shi Y, Di Giammartino DC, Taylor D, Sarkeshik A, Rice WJ, Yates JR III, Frank J and Manley JL: Molecular architecture of the human pre-mRNA 3' processing complex. Mol Cell 33: 365-376, 2009.

22. Masamha CP, Xia Z, Yang J, Albrecht TR, Li M, Shyu AB, Li W and Wagner EJ: CFIm25 links alternative polyadenylation to glioblastoma tumour suppression. Nature 510: 412-416, 2014.

23. Elkon R, Drost J, van Haaften G, Jenal M, Schrier M, Oude Vrielink JA and Agami R: E2F mediates enhanced alternative polyadenylation in proliferation. Genome Biol 13: R59, 2012.

24. Lee RC, Feinbaum RL and Ambros V: The C. elegans heterochronic gene lin-4 encodes small RNAs with antisense complementarity to lin-14. Cell 75: 843-854, 1993.

25. Pasquinelli AE, Reinhart BJ, Slack F, Martindale MQ, Kuroda MI, Maller B, Hayward DC, Ball EE, Degnan B, Müller P, et al: Conservation of the sequence and temporal expression of let-7 heterochronic regulatory RNA. Nature 408: 86-89, 2000.

26. Reinhart BJ, Slack FJ, Basson M, Pasquinelli AE, Bettinger JC Rougvie AE, Horvitz HR and Ruvkun G: The 21-nucleotide let-7 RNA regulates developmental timing in Caenorhabditis elegans. Nature 403: 901-906, 2000.

27. Kobayashi E, Satow R, Ono M, Masuda M, Honda K, Sakuma T, Kawai A, Morioka H, Toyama Y and Yamada T: MicroRNA expression and functional profiles of osteosarcoma. Oncology 86 94-103, 2014
28. Osaki M, Takeshita F, Sugimoto Y, Kosaka N, Yamamoto Y, Yoshioka Y, Kobayashi E, Yamada T, Kawai A, Inoue T, et al: MicroRNA-143 regulates human osteosarcoma metastasis by regulating matrix metalloprotease-13 expression. Mol Ther 19: 1123-1130, 2011.

29. Duan Z, Choy E, Harmon D, Liu X, Susa M, Mankin H and Hornicek F: MicroRNA-199a-3p is downregulated in human osteosarcoma and regulates cell proliferation and migration. Mol Cancer Ther 10: 1337-1345, 2011

30. Kloosterman WP and Plasterk RH: The diverse functions of microRNAs in animal development and disease. Dev Cell 11: 441-450, 2006

31. Meng F, Henson R, Wehbe-Janek H, Ghoshal K, Jacob ST and Patel T: MicroRNA-21 regulates expression of the PTEN tumor suppressor gene in human hepatocellular cancer. Gastroenterology 133: 647-658, 2007.

32. Jovanovic $M$ and Hengartner MO: miRNAs and apoptosis: RNAs to die for. Oncogene 25: 6176-6187, 2006.

33. Lu J, Getz G, Miska EA, Alvarez-Saavedra E, Lamb J, Peck D, Sweet-Cordero A, Ebert BL, Mak RH, Ferrando AA, et al: MicroRNA expression profiles classify human cancers. Nature 435: 834-838, 2005.

34. Esquela-Kerscher A and Slack FJ: Oncomirs-microRNAs with a role in cancer. Nat Rev Cancer 6: 259-269, 2006.

35. Calin GA and Croce CM: MicroRNA signatures in human cancers. Nat Rev Cancer 6: 857-866, 2006.

36. Deng G, Hu C, Zhu L, Huang F, Huang W, Xu H and Nie W: Downregulation of ROS-FIG inhibits cell proliferation, colony-formation, cell cycle progression, migration and invasion, while inducing apoptosis in intrahepatic cholangiocarcinoma cells. Int J Mol Med 34: 661-668, 2014.

37. Zhang ZG, Niu XY, He XJ and Shu J: Ginsenoside Rg1 reduces toxicity of fine particulate matter on human alveolar epithelial cells: A preliminary observation. Mol Med Rep 9: 989-992, 2014.

38. Tsujimoto $M$, Doi $T$, Kuroyanagi G, Yamamoto $N$, Matsushima-Nishiwaki R, Iida Y, Enomoto Y, Iida H, Ogura S, Otsuka $\mathrm{T}$, et al: $\alpha \mathrm{B}$-crystallin reduces ristocetin-induced soluble CD40 ligand release in human platelets: Suppression of thromboxane A2 generation. Mol Med Rep 12: 357-362, 2015.

39. Zhang F, Li ZL, Xu XM, Hu Y, Yao JH, Xu W, Jing HR, Wang S, Ning SL, Tian XF: Protective effects of icariin-mediated SIRT1/FOXO3 signaling pathway on intestinal ischemia/reperfusion-induced acute lung injury. Mol Med Rep 11: 269-276, 2015.

40. Bekker-MéndezC,Guzmán-AguilarRM,Hernández-Cueto MA Huerta-Yepez S, Jarillo-Luna RA, González-Veyrand E and González-Bonilla CR: TUNEL-positive cells in the surgical border of an amputation due to infected diabetic foot. Mol Med Rep 5: 363-372, 2012.

41. Ji F, Zhang H, Wang Y, Li M, Xu W, Kang Y, Wang Z, Wang Z, Cheng P, Tong D, et al: MicroRNA-133a, downregulated in osteosarcoma, suppresses proliferation and promotes apoptosis by targeting Bcl-xL and Mcl-1. Bone 56: 220-226, 2013.

42. Zhang H, Cai X, Wang Y, Tang H, Tong D and Ji F: microRNA-143, down-regulated in osteosarcoma, promotes apoptosis and suppresses tumorigenicity by targeting Bcl-2. Oncol Rep 24: 1363-1369, 2010.

43. Zhu S, Wu H, Wu F, Nie D, Sheng S and Mo YY: MicroRNA-21 targets tumor suppressor genes in invasion and metastasis. Cell Res 18: 350-359, 2008.

44. Zhu S, Si ML, Wu H and Mo YY: MicroRNA-21 targets the tumor suppressor gene tropomyosin 1 (TPM1). J Biol Chem 282: 14328-14336, 2007.

45. Gorlick R: Current concepts on the molecular biology of osteosarcoma. Cancer Treat Res 152: 467-478, 2009.

46. Bacci G, Bertoni F, Longhi A, Ferrari S, Forni C, Biagini R, Bacchini P, Donati D, Manfrini M, Bernini G and Lari S: Neoadjuvant chemotherapy for high-grade central osteosarcoma of the extremity. Histologic response to preoperative chemotherapy correlates with histologic subtype of the tumor. Cancer 97: 3068-3075, 2003.

47. Jianwei Z, Fan L, Xiancheng L, Enzhong B, Shuai L and Can L: MicroRNA 181a improves proliferation and invasion, suppresses apoptosis of osteosarcoma cell. Tumour Biol 34: 3331-3337, 2013. 